\title{
Novel Planar Electromagnetic Bandgap Structures for Mitigation of Switching Noise and EMI Reduction in High-Speed Circuits
}

\author{
Jie Qin, Omar M. Ramahi, and Victor Granatstein, Life Member, IEEE
}

\begin{abstract}
Planar electromagnetic bandgap (EBG) structures with novel meandered lines and super cell configuration are proposed for mitigating simultaneous switching noise propagation in high-speed printed circuit boards. An ultrawide bandgap extending from $250 \mathrm{MHz}$ to $12 \mathrm{GHz}$ and beyond is demonstrated by both simulation and measurement, and a good agreement is observed. These perforated EBG-based power planes may cause spurious and unwanted radiation. In this paper, leakage radiation through these imperfect planes is carefully investigated. It is found that the leakage field from these planar EBG structures is highly concentrated around the feed point, and the field intensity is attenuated dramatically when passing across several periods of patches. A novel concept of using these EBG structures for electromagnetic interference reduction is also introduced. Finally, the impact of power plane with EBG-patterned structures on signal integrity is studied.
\end{abstract}

Index Terms-Electromagnetic bandgap (EBG), electromagnetic interference (EMI), signal integrity (SI), simultaneous switching noise (SSN).

\section{INTRODUCTION}

A $\mathrm{N}$ IDEAL power distributed system (PDS) is assumed to supply clean power to integrated circuits. However, electromagnetic noise in power/ground planes can cause fluctuation or disturbance in the power supply voltage, which, in turn, leads to false switching and malfunctioning in digital or analog circuit. With the progress of modern CMOS technology, especially the increase in clock frequency and pulse edge rate, and the decrease in power supply voltage and noise margin, the power/ground noise creates significant and new challenges for electromagnetic interference/electromagnetic compatibility (EMI/EMC) and packaging engineers. Simultaneous switching noise (SSN) has become one of the major concerns [1], [2]. This type of electromagnetic disturbance (noise), also known as delta-I noise or power/ground plane bounce, has been discussed intensively over the last decade and different approaches have been used to maintain a noise-free PDS. Most prominent of these involve the use of discrete decoupling capacitor and embedded capacitance [3], [4]. However, the method of decoupling capacitors fails when operated at high frequency due to the inherent lead inductance of capacitors. Embedded capacitance,

Manuscript received August 18, 2006; revised December 4, 2006 and February 14, 2007.

J. Qin and V. Granatstein are with the Department of Electrical and Computer Engineering, University of Maryland, College Park, MD 20742 USA (e-mail: cyd@mail.umd.edu; vlg@glue.umd.edu).

O. M. Ramahi is with the Department of Electrical Engineering, University of Waterloo, Waterloo, ON N2L 3G1 Canada (e-mail: oramahi@uwaterloo.ca).

Digital Object Identifier 10.1109/TEMC.2007.902193 on the other hand, is an expensive solution and reliability considerations limit its practical use. Furthermore, the embedded capacitance does not eliminate higher order resonant modes. The question that remains unanswered is: Are there alternative or complementary solutions for mitigating power planes noise over wider frequency range?

Electromagnetic bandgap (EBG) structures, proposed in recent years, have proven effective for noise suppression at frequencies above $1 \mathrm{GHz}$, which the methods mentioned above cannot achieve. EBG structure is a kind of periodic structure that can form high-impedance surface (HIS) to prevent the propagation of electromagnetic wave over some frequency range. It was first proposed by Sievenpiper for antenna application in order to suppress surface wave [5], [6]. Later, this concept of using EBG to suppress surface wave has inspired other authors to use this structure for suppressing noise in power planes [7]-[12]. The earlier structures used three layers where the EBG pattern layer with specially designed via is inserted between the power plane and the ground plane, which makes the fabrication more expensive. Recently, new planar EBG structures were reported for switching noise mitigation as in [13]-[15], and for isolation in mixed signal boards as in [16]. These new structures consist of a two-layer power distribution system with one of the layers patterned in a periodic fashion, effectively creating a frequency filtering or EBG effect. These novel structures, in sharp contrast to previous multilayer EBG structures used for power plane noise mitigation, do not have vias. These features make such structures very attractive from the manufacturing and cost perspectives.

In this paper, we present planar EBG-patterned two-layer PCBs used for noise mitigation. The drawback of earlier structures is the limited width of the bandgap. We show that by introducing novel structures with meander lines in conjunction with the concept of a supercell, it is possible to not only extend the bandgap beyond what was achieved in previous works, but also decrease the lower edge of the bandgap to approximately $250 \mathrm{MHz}$ without increasing the EBG patch size. The designs presented here can eliminate the decoupling capacitors typically used in the subgigahertz region.

While planar EBG structures offer clear advantages in comparison to multilayer structures with or without vias, it is important to pay careful attention to the possibility of electromagnetic leakage through the perforated layer. In fact, in practical scenarios, as recent studies have shown (see [17]-[20]), the perforated power plane can lead to increased radiation from the PCB. While previous works focused on the far-field radiation arising from 
the use of planar EBG structures [17], this paper considers the effect of planar structure on the near field in order to understand the potential for electromagnetic interference within the PCB itself. Moreover, this paper introduces the novel concept of applying the planar EBG power plane as a shield to reduce EMI coming from power busses.

This paper is organized as follows. Section II introduces the novel designs of EBG structure with meander lines and supercell, and their performance is characterized by scattering parameter through both simulation and measurement. In Section III, the leakage radiation through these perforated planes is investigated, and a concept of EMI reduction using these EBG-patterned surfaces is examined. In Section IV, the impact of these EBG-patterned power planes on signal integrity (SI) is discussed, and a differential signaling method is introduced to improve the SI. Finally, conclusions are drawn in Section V.

\section{Planar EBG Structure Designs And SSN Mitigation}

\section{A. Structure Design}

Fig. 1(a) shows the proposed two-layer power/ground plane with meander line EBG structure. The whole dimension of this structure with $3 \times 5$ unit cells is $90 \mathrm{~mm} \times 150 \mathrm{~mm}$. The solid layer can be used for one voltage level, and the EBG-patterned layer is used for the second voltage level. Between these two layers, a uniform substrate material FR-4 with dielectric constant 4.4 and layer thickness of $1.54 \mathrm{~mm}$ is selected due to its manufacturing flexibility at our local facilities.

The schematics of the unit cell of size $30 \mathrm{~mm} \times 30 \mathrm{~mm}$, and its corresponding parameters are shown in Fig. 1(b). The distance between the centers of adjacent patches is $30 \mathrm{~mm}$, the patch width is $28 \mathrm{~mm}$, and the gap between the neighboring patches is $2 \mathrm{~mm}$. The width of the meandered line is $0.2 \mathrm{~mm}$. For one-dimensional wave propagation, the unit cell of this Meander-L EBG structure can be modeled with the equivalent circuit as shown in Fig. 1(c). This qualitative model is inspired by the physical behavior of the fields in the patch. It consists of two parts. The first part describes the propagation characteristics between the EBG patch and the continuous power plane, represented by equivalent inductance $L_{p}$ and capacitance $C_{p}$. The second part characterizes the bridge effects between two adjacent unit cells, where the gap between the two neighboring unit cells induces the fringe capacitance $C_{b}$, and the bridge connecting the neighboring unit cells as the inductor $L_{b}$. Thus, this EBG structure can be conceptually viewed as an electrical filter of parallel LC resonator.

The center frequency of the stop-band for the EBG structure can be expressed semiquantitatively as $f_{0}=1 /\left(2 \pi \sqrt{ }\left(L_{b} C_{b}\right)\right)$. Consequently, one observes that in order to effectively implement the EBG structure in the lower frequency region, a longer bridge length corresponding to an increase in inductance would be required. Therefore, we introduce a meander line as the connecting bridge ( $\mathrm{dc}$ link) between adjacent patches. The width of the meandered line is $0.2 \mathrm{~mm}$, which is within the fabrication tolerance capability of our laboratory facility. (The LPKF prototype machine was used for fabricating the prototypes discussed in this paper.)

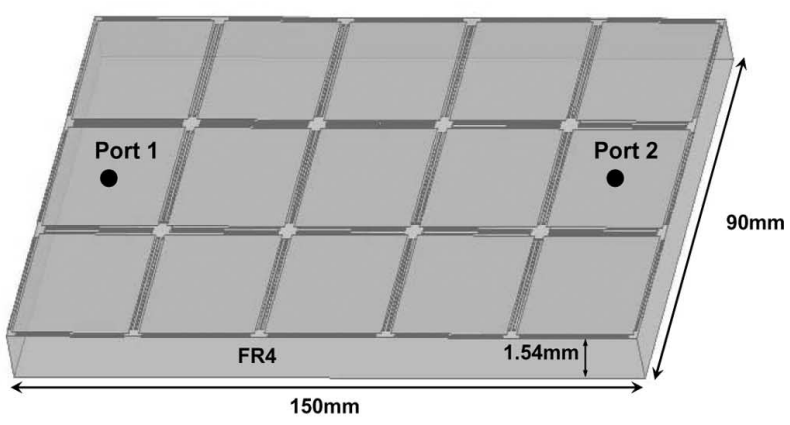

(a)

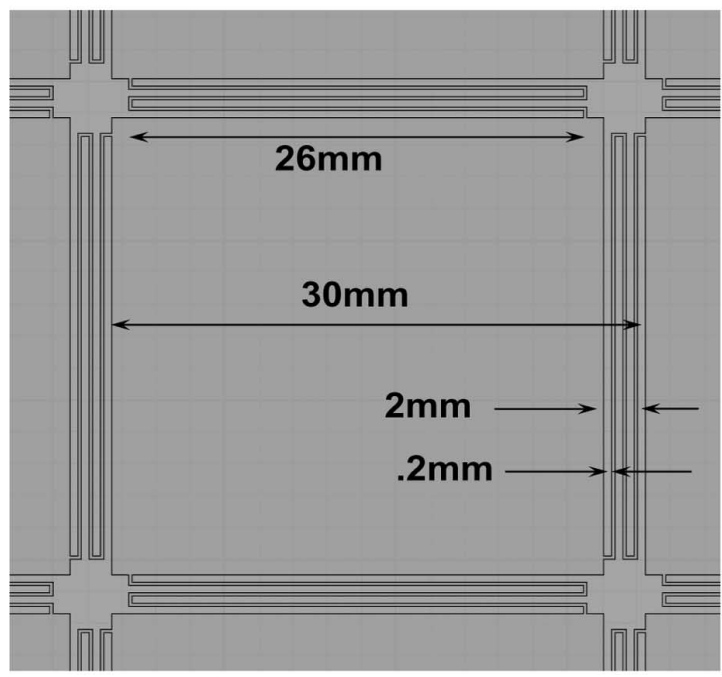

(b)

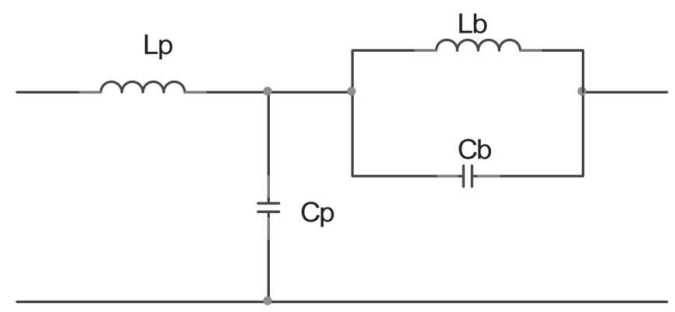

(c)

Fig. 1. (a) Planar EBG structure with Meander-L bridge showing the location of the ports used for $S_{21}$ parameter measurements. (b) Unit cell showing the meander line bridge and dimensions. (c) Qualitative equivalent circuit model for unit cell of the Meander-L EBG structure.

\section{B. Simulation and Experimental Result}

Performance of the EBG structures is characterized by S-parameters measured with the Agilent E8364A vector network analyzer (VNA). Each test port is located at the patch center as shown in Fig. 1(a). In Fig. 2, we show the simulation result of the EBG surface with meandered lines as obtained from the Ansoft High Frequency Structure Simulator (HFSS) [21] finiteelement based algorithm along with the measured data. HFSS is a three-dimensional finite-element-based electromagnetic analysis software that uses tangential field tetrahedron elements for added accuracy. (For a comprehensive background on the finiteelement method, the reader is referred to [22] and references therein.) The data show that an ultrawide bandgap is achieved using this new structure with strong agreement between simulation 


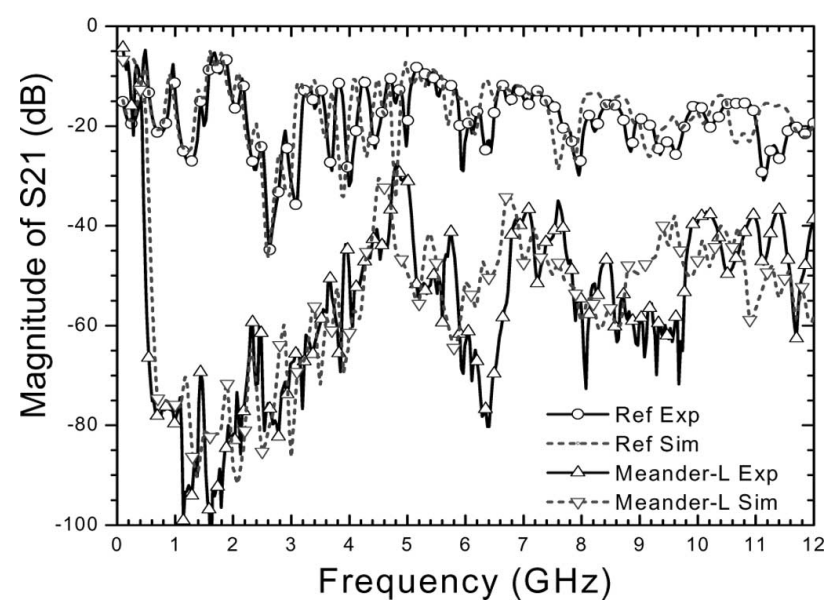

Fig. 2. Numerical simulation and measurement result for magnitude of $S_{21}$.

and measurements. The stopband frequency range is observed to extend from approximately $450 \mathrm{MHz}$ to $12 \mathrm{GHz}$ and beyond. The definition of the bandwidth adopted here is the continuous frequency range over which the magnitude of the $S_{21}$ is maintained below $-28 \mathrm{~dB}$. (There is no standard definition for suppression bandwidth in the context of switching noise as the degree of suppression is application specific. Here, the $-28 \mathrm{~dB}$ was chosen for convenience as it represents significant suppression in comparison to the reference case.)

Next, we introduce the concept of a supercell. The detail mechanism of this structure is discussed in [15]. The basic idea is to create a cell comprising two patches with different topology. The new supercell will then be cascaded resulting in a new structure that is expected to embody the bandgaps arising from the use of each of the two topologies if they were used separately, in addition to the bandgap arising from the periodicity formed by this new supercell. Fig. 3(a) shows the basic unit of this supercell structure. It shows adjacent EBG patches with two different connecting bridge topologies: a straight line and a meander line. The patch is kept at the same size of $30 \mathrm{~mm} \times 30 \mathrm{~mm}$. The fabricated PCB with an overall dimension of $90 \mathrm{~mm} \times 150 \mathrm{~mm}$ is shown in Fig. 3(b).

The $S_{21}$ parameter data (simulation and measurement) is shown in Fig. 4, where we observe not just an appreciably wide bandgap but, more importantly, the shift of the lower edge of the stopband downward to approximately $250 \mathrm{MHz}$. This bandwidth improvement realized by the supercell can lead to cost reduction by eliminating the decoupling capacitors needed to maintain minimal noise in the sub-500-MHz range.

\section{RADIATION ANALYSIS}

\section{A. Leakage Radiation Through EBG-Patterned PCB}

Previous studies show that the radiation from solid power planes comes from a time-varying electric field at the board edges similar to the way a patch antenna radiates [23], [24]. If the signal is fed at one port and received at another port, part of the energy radiates at the edge of the board while never reaching any of the other ports. The radiation loss can be characterized by the quantity $1-\left|S_{11}\right|^{2}-\left|S_{21}\right|^{2}$. This is different from a closed

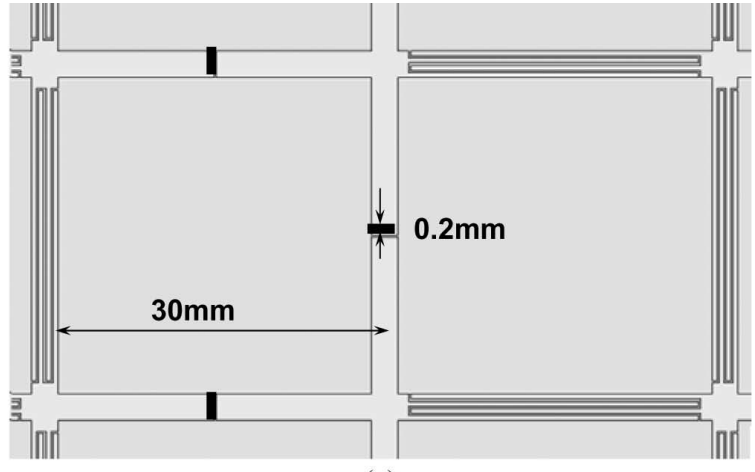

(a)

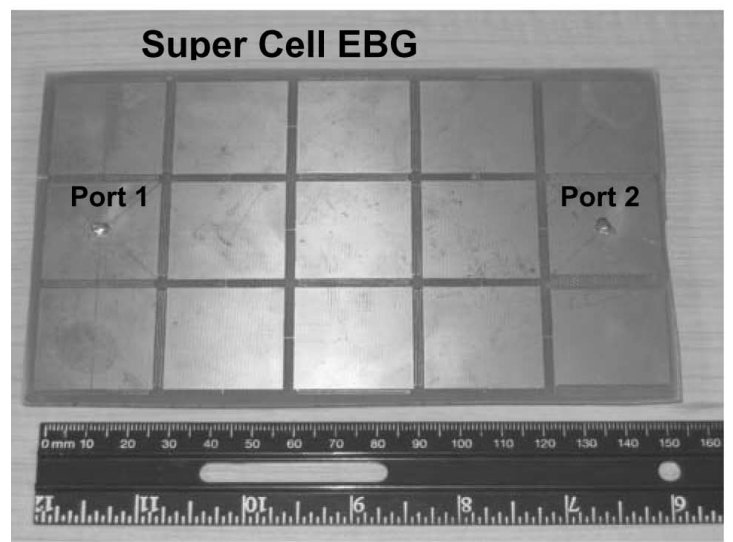

(b)

Fig. 3. (a) Schematic top view of the supercell with dimensions. (b) Whole structure of fabricated PCB showing test ports for S parameter measurement.

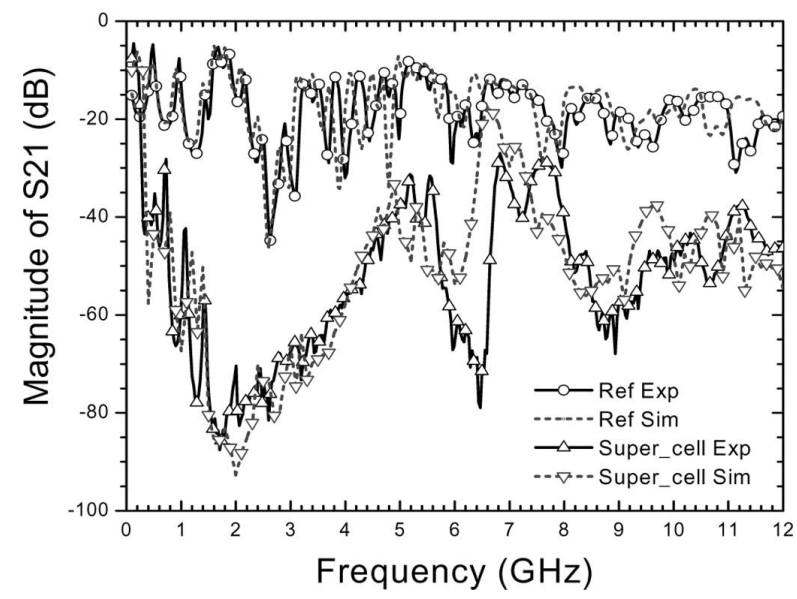

Fig. 4. Numerical simulation and measurement of the magnitude of $S_{21}$ for the novel structure proposed in this paper (supercell) and for the two-layer power plane.

lossless system, in which the total combination of reflection coefficient and transmission coefficient equals to unity. For the perforated planar EBG structures described earlier, in addition to the radiation at the edges of board, there are gaps between the cells, which may cause extra radiation leakage, especially at the frequency where the patches resonate. In sharp contrast to the three- or multilayer EBG structures with solid power plane, this extra radiation from these perforated boards can potentially 


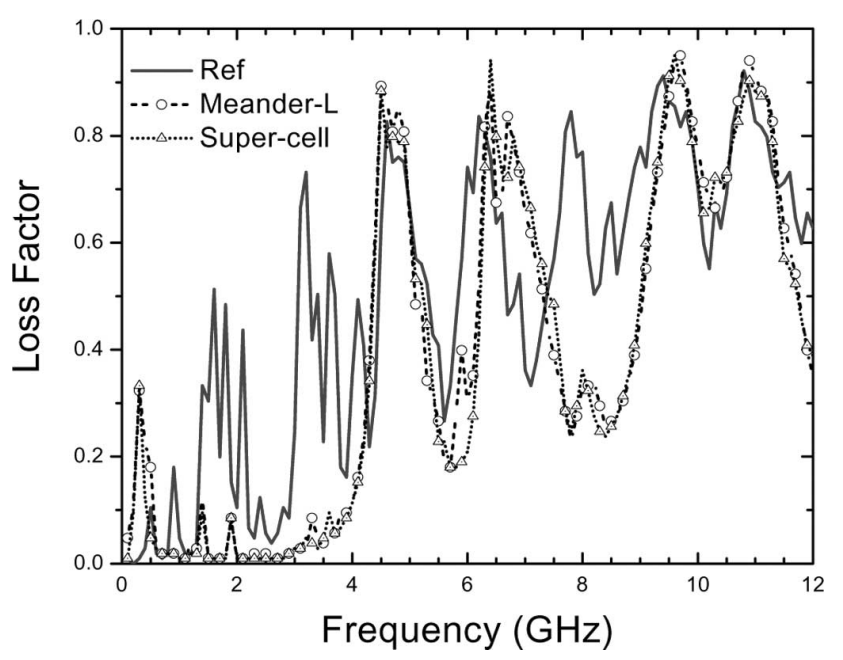

Fig. 5. Radiation loss for different structures.

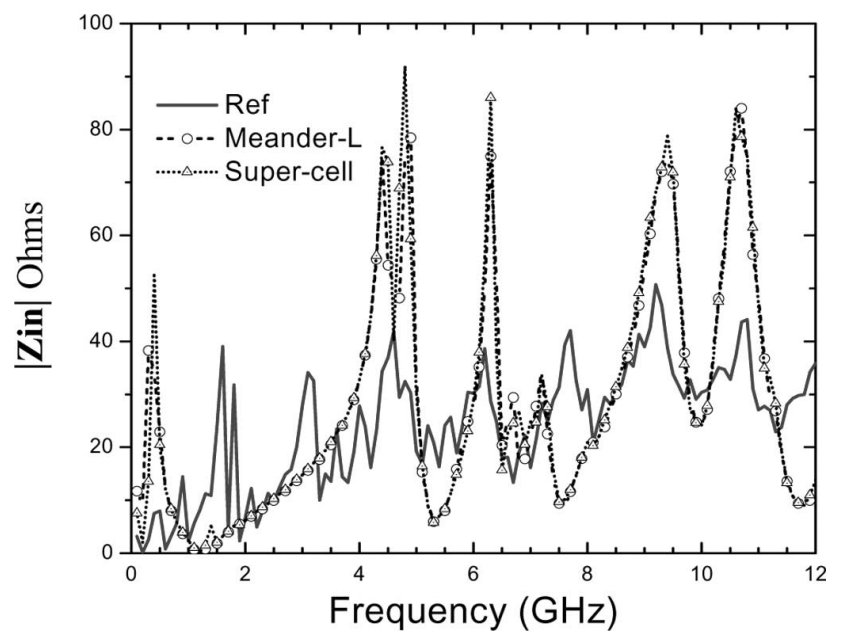

Fig. 6. Simulated port input impedance for different structures.

be sufficient to create incompatibility with either circuit functionality, or simply be high enough to violate regulatory radiation standards.

Here, the calculated loss factor $1-\left|S_{11}\right|^{2}-\left|S_{21}\right|^{2}$ is examined for EBG-based power planes and compared to the case with solid power plane. The results shown in Fig. 5 demonstrate that the radiation peaks at 480, $960 \mathrm{MHz}$, etc., which arise from the cavity modes of the $90 \mathrm{~mm} \times 150 \mathrm{~mm}$ reference board, are significantly suppressed for the EBG-patterned structures. The strong radiation peaks at $4.7,6.6,9.4$, and $10.6 \mathrm{GHz}$ for the EBG-patterned power planes are due to the patch resonance, which can be further confirmed from the input impedance (self impedance of excitation port) data shown in Fig. 6, where the first high impedance peak corresponds to the $f_{20,02}$ modes of the $30 \mathrm{~mm} \times 30 \mathrm{~mm}$ patch at $4.7 \mathrm{GHz}$ followed by even numbered modes $f_{22}, f_{40}$. Notice that odd-numbered modes do not appear because the feed port consists of a center-fed patch [25]. When the patch is fed off center, the odd-numbered modes appear consistent with the cavity resonance behavior (results are not shown for brevity).

However, the radiation loss from these impedance peaks does not show much increase when comparison is made to the case

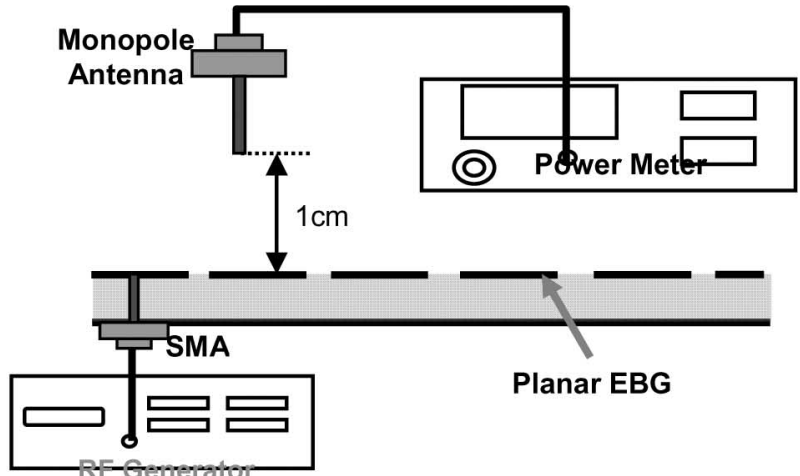

(a)

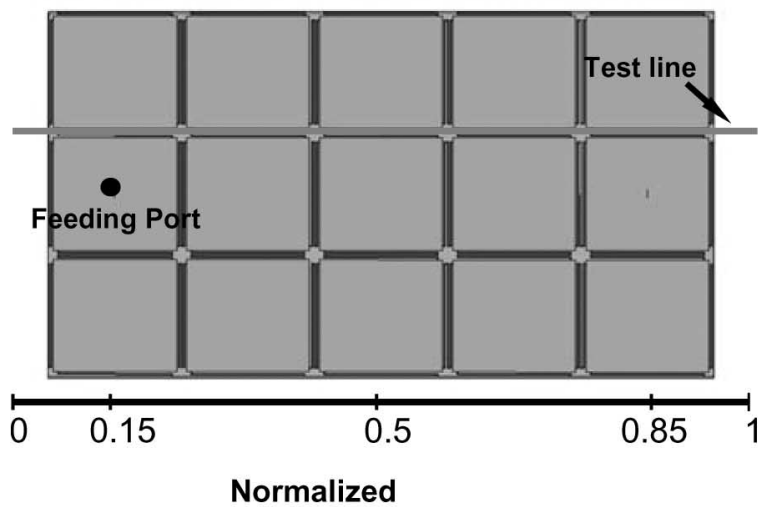

(b)

Fig. 7. Near-field measurement setup.

of solid reference plane. This is due to the resonance modes overlap between those of the reference plane and of the single patch. Overall, the data show that the total radiated power from the EBG-patterned PCB is diminished, especially at the lower frequency region (below the fundamental resonance of patch at 4.7 $\mathrm{GHz}$ ) in comparison to the reference plane.

We emphasize here that the measure of the radiation loss used here, i.e., the quantity $1-\left|S_{11}\right|^{2}-\left|S_{21}\right|^{2}$, is only indicative of the fact that the electromagnetic energy prevented from propagating between the two ports is either stopped at the source (the transmitting port, due to a change in its input impedance as a result of using the EBG patches) or leaks through other channels. A more detailed analysis of the power leakage is given in Section III-B.

\section{B. Near-Field Characterization}

In order to obtain a better understanding of the shielding behavior of perforated power planes, the near field of these proposed planar EBG structures is studied. In all the cases studied here, comparison is always made to the fields arising from a two-layer solid power plane.

The analysis here is carried out by using a monopole antenna connected to a power meter to monitor the field along a test line positioned $1 \mathrm{~cm}$ above the EBG-patterned plane and aligned along the open slot of the EBG patches extending from the left to the right edge (represented by normalized distance) as shown in Fig. 7(a) and (b). An RF signal of $10 \mathrm{dBm}$ is launched into the 


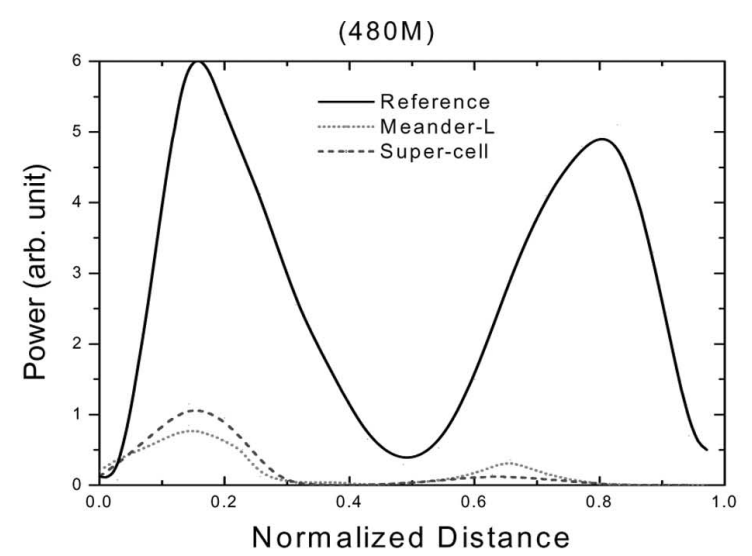

(a)

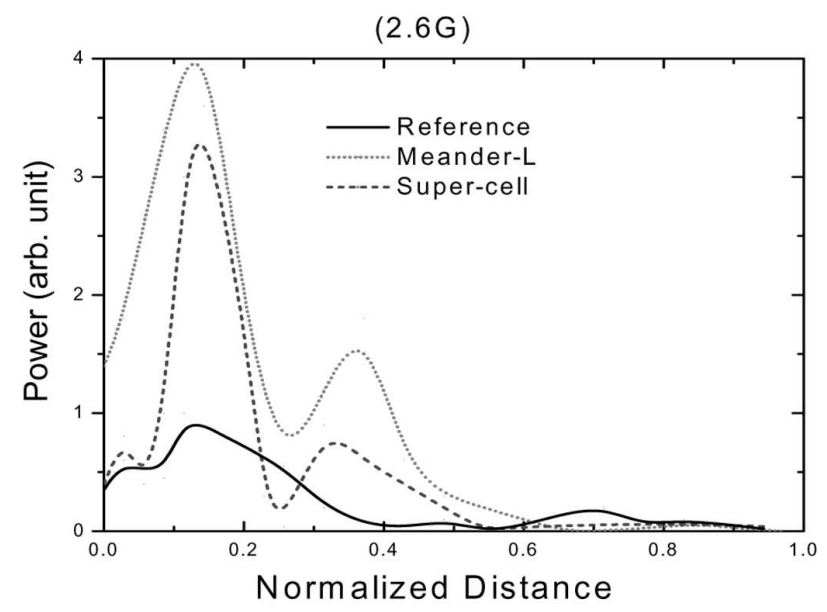

(b)

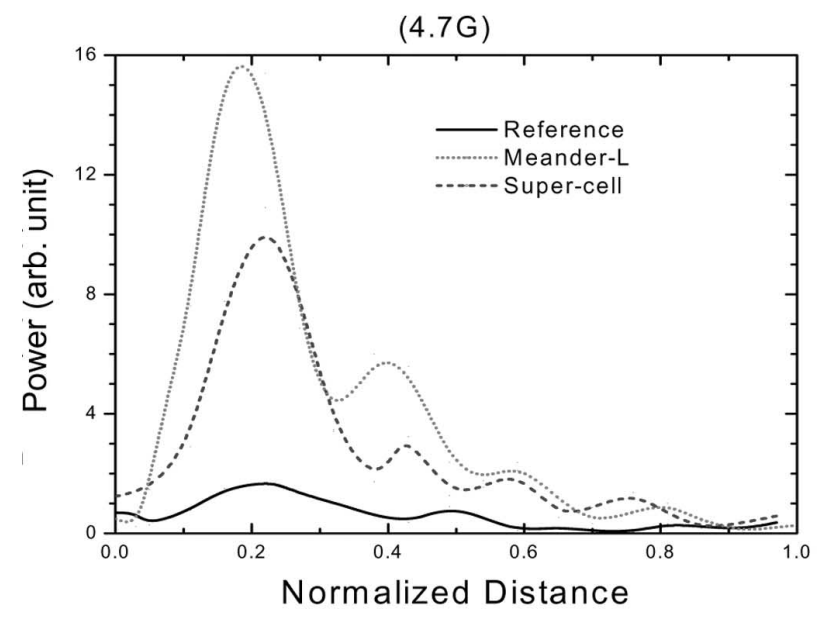

(c)

Fig. 8. Near-field radiation intensity measured $1 \mathrm{~cm}$ above the board over the test line shown in Fig. 7. Measurements performed for three different frequencies. (a) $480 \mathrm{MHz}$. (b) $2.6 \mathrm{GHz}$. (c) $4.7 \mathrm{GHz}$.

power plane through an SMA-type connector at the feeding port shown in Fig. 7. The field intensity as a function of normalized distance is shown in Fig. 8(a)-(c) for different frequencies. The frequencies selected here are $480 \mathrm{MHz}$ (fundamental resonance frequency for reference board), $2.6 \mathrm{GHz}$ (within the stopband of the EBG structure), and $4.7 \mathrm{GHz}$ (patch fundamental resonant frequency).

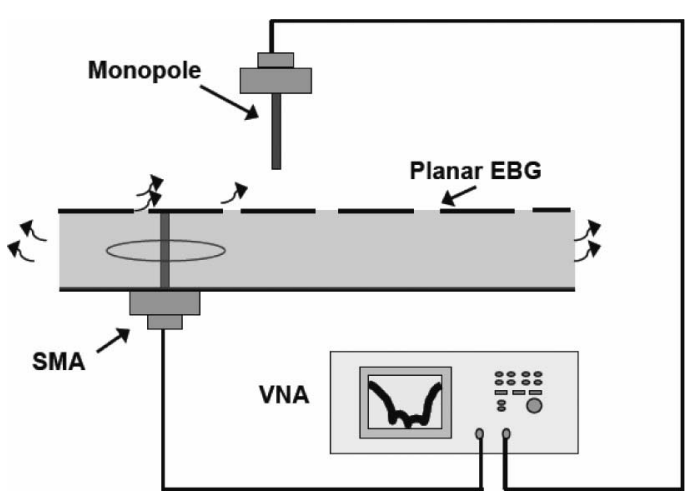

Fig. 9. Experimental setup for $\mathrm{S}_{21}$ measurement for testing EMI shielding.

In comparison to the solid reference plane, at the resonance frequency of the PCB at $480 \mathrm{MHz}$, the data show that the near field is significantly suppressed by using the EBG structures. We note that while the $480 \mathrm{MHz}$ is at the bottom edge of the stopband, it is a resonant mode for the solid plane (reference). The reason for the reduced near field is attributed to the perforated top plane which prevents the "dipole-like" behavior that is characteristic of solid planes (or patch antennas). Therefore, the planar EBG structure has the added advantage of reducing the dominant resonance of the board.

At the 2.6- and 4.7-GHz frequencies, the field leakage is stronger when using the planar EBG structure in comparison to the solid plates PCB. In fact, we observe the field to be highly concentrated around the feed port. This is expected at these frequencies as the EBG blocks the transmission of energy between the ports, and one expects a high reflection at the transmitting port and, hence, the higher concentration of the field there. Furthermore, we observe that the field intensity gradually dies down after traversing several EBG patches. This attenuation is mainly attributed to the EBG structure surface current mitigation mechanism.

\section{EMI Shielding Using EBG-Patterned Surface}

The results obtained above demonstrate the distinct features of EBG-patterned structures such as reducing the total radiation, suppressing the resonance modes and surface current propagation, and localizing the field leakage around the excitation region. This suggests the use of the EBG-patterned surface as an electromagnetic shield to reduce the radiation from power busses of PCBs.

Fig. 9 shows the experimental setup used to test the shielding effectiveness of the EBG planar structure. The total amount of radiation from both the edges of the board and open slots is measured by $S_{21}$ as a representative for the radiated power at various test points along the test line as shown in Fig. 7(b). Here, the input signal is fed to the parallel-plate environment through an SMA connector at one port of VNA. A monopole probe is connected to the other port of the VNA representing the receiver. Fig. 10(a)-(d) shows the measured magnitude of the $S_{21}$ corresponding to different normalized distances of 0.15 (source), 0.44, 0.73, and 1 (far end of board edge). A 


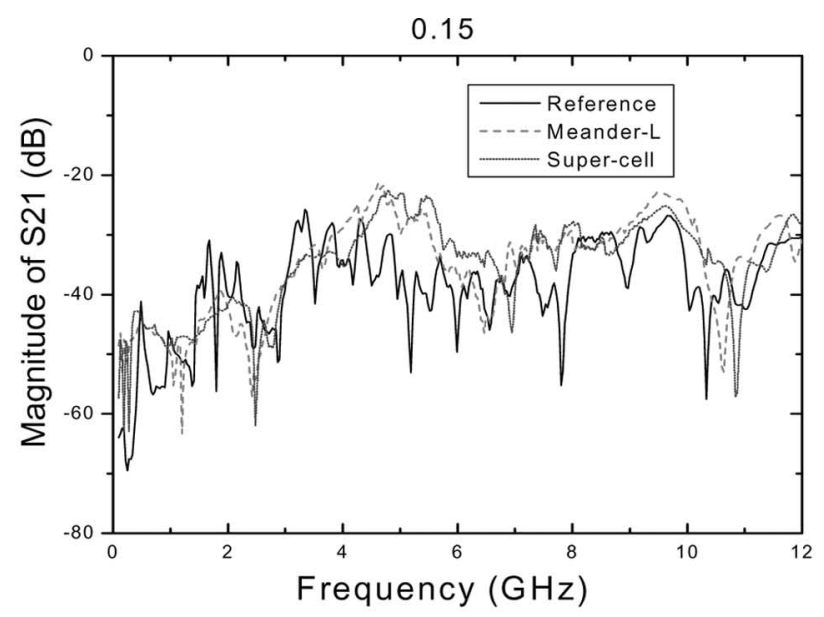

(a)

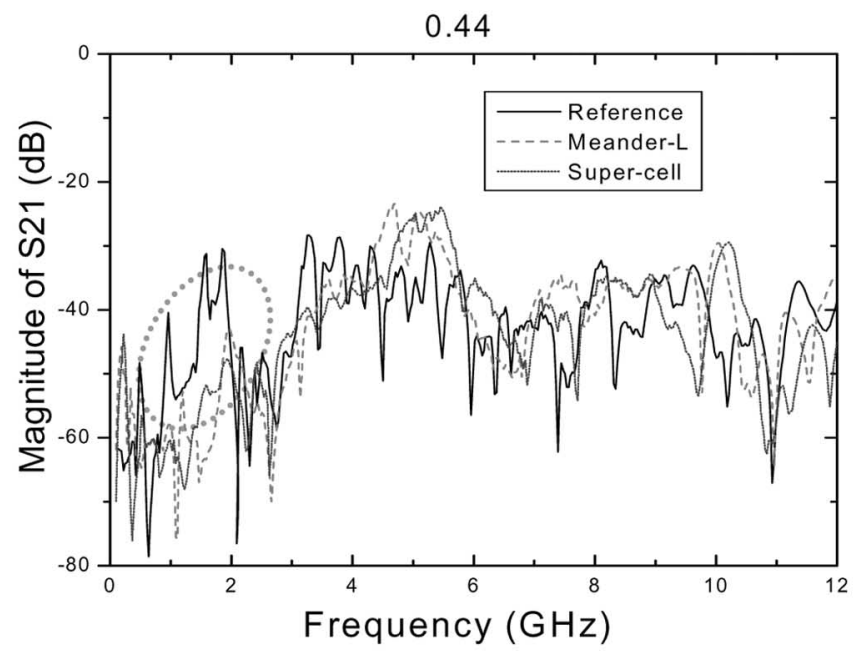

(b)

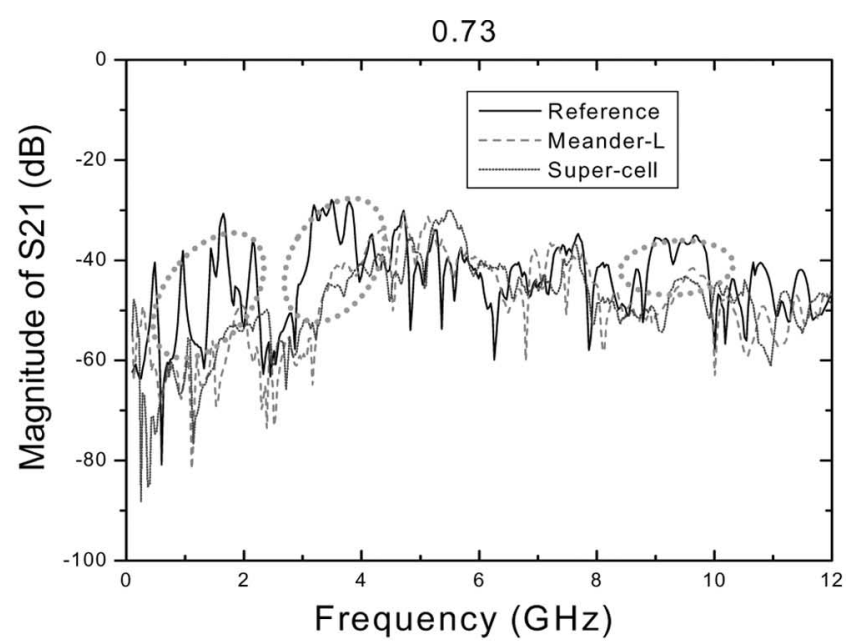

(c)

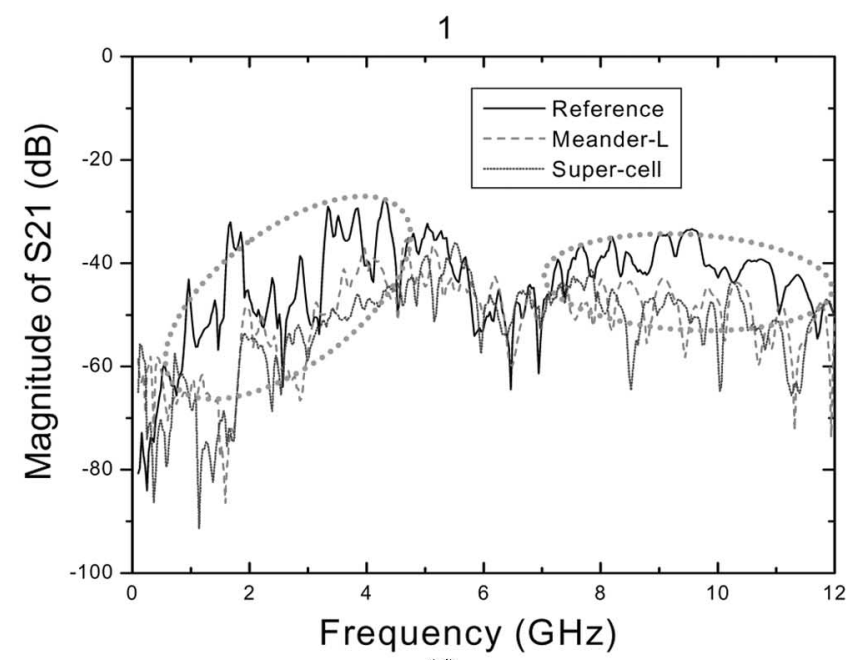

(d)

Fig. 10. $S_{21}$ parameter measured at different test point represented by normalized distance (with respect to the size of the board). (a) 0.15 (coinciding with the source location). (b) 0.44. (c) 0.75. (d) 1 .

comparison is made with the reference structure in which both top and bottom layers are made of solid metal. The data clearly demonstrate that for planar EBG-based power planes, when the test point is away from the excitation region, wider and deeper bandgap can be achieved. Near-field intensity with average suppression of $20 \mathrm{~dB}$ is achieved in a wide frequency range from 0.5 to $4.6 \mathrm{GHz}$ and 7 to $12 \mathrm{GHz}$ in comparison to the case without EBG structures. It is important to note, however, that such suppression is achieved at a distance spanning a few patches from the source in a manner fully consistent with the filter theory and EBG surface current suppression mechanism.

\section{IMPACt OF EBG Power Plane ON SI}

Although the proposed EBG-patterned power plane shows good performance on noise mitigation and corresponding EMI reduction at a wide frequency range, its effect on the propagating signal needs to be analyzed, especially in the light of recent studies [14], [26], [27].
In this section, the signal integrity for the proposed EBG structures is investigated in the time domain using the eye diagram. The eye diagram, which is commonly used in SI analysis, gives an indication of the signal quality; the larger the eye opening, the better the signal quality.

\section{A. Single-Ended Signal}

Here, we consider four-layer PCB structures and compare two different cases. In the first case, the signal propagates on a microstrip line and is referenced (return current path) to a solid plane (structure A). In the second case, the signal is referenced to an EBG-patterned plane (structure B). For structure A, the middle two layers serve as solid power/ground planes. The signal propagates along the trace on the top signal layer, through via down to the bottom layer and back to the top layer again. All the layers are filled with FR4, with thickness of $1.54 \mathrm{~mm}$ for each layer. The board geometry is $90 \mathrm{~mm} \times 150 \mathrm{~mm}$ as used in previous examples. The signal traces are designed as $50-\Omega$ lines (with respect to a solid reference plane). In structure B, 


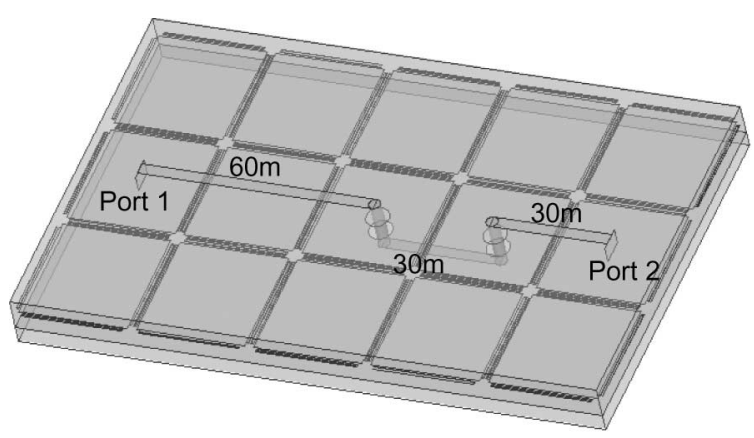

(a)

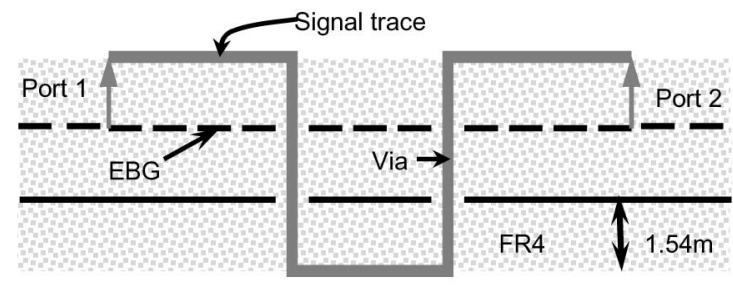

(b)

Fig. 11. Four-layer PCBs with signal layer referring to the Meander-L EBGpatterned power plane. (a) Three-dimensional view. (b) Side-view.

the middle two layers are replaced with a meander line EBG structure. The 3-D view and side view of structure B are shown in Fig. 11(a) and (b), respectively. (No illustration for structure A is shown for brevity.)

A nonreturn to zero (NRZ), pseudorandom binary sequence (PRBs) $2^{7}-1$ is sent at the input (port 1), and the signal propagation properties are monitored at the output (port 2). The launched PRBs are coded at $1 \mathrm{~Gb} / \mathrm{s}$, with $1-\mathrm{V}$ swing, and nominal rise/fall time is $200 \mathrm{ps}$. The eye patterns are generated by using Agilent ADS convolution simulator, which convolves the inverse fast Fourier transforms (IFFTs) of the scattering parameter along the traces directly. The simulated eye diagrams for the signal streams at the end of the traces of structures $A$ and $B$ are shown in Fig. 12(a) and (b). The vertical thickness of the line in the eye diagram is indicative of the ac voltage noise, whereas the horizontal thickness is indicative of the ac timing noise (jitter).

It is seen that the signal quality is seriously degraded for structure B. This is mainly due to the strong reflections arising from these imperfect EBG-patterned power planes.

\section{B. Differential Pair}

In order to improve the signal quality, differential signaling approach is adopted. Differential signaling has a number of advantages over single-ended signaling. Coupling between the two signal lines allows differential signals to propagate with less noise picked up from other active nets. The greater the coupling, the more robust the differential signals will be to discontinuities and imperfections. In the example considered here, the traces are designed with a differential impedance of $100 \Omega$ for the differential pair configuration shown in Fig. 13(a). The width of the lines is $2 \mathrm{~mm}$, and the spacing between the

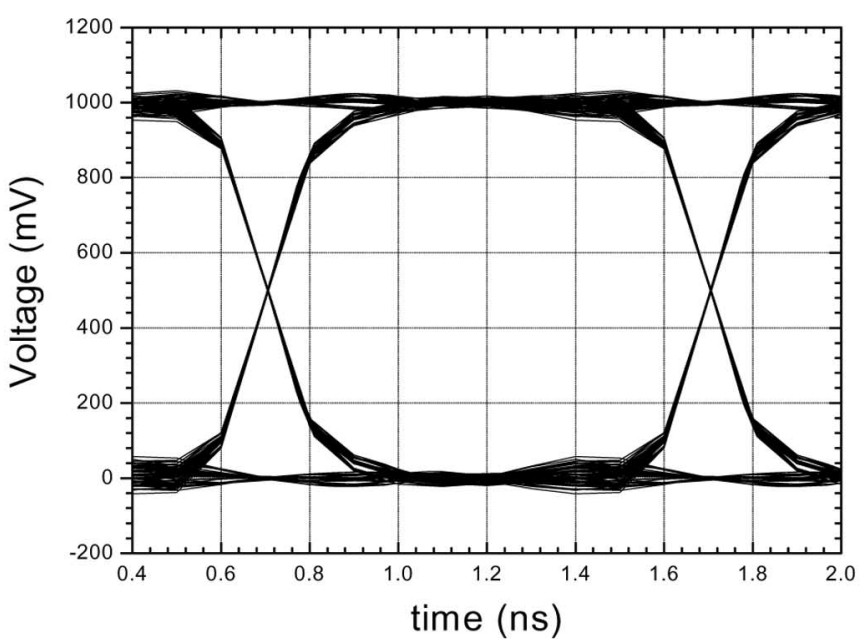

(a)

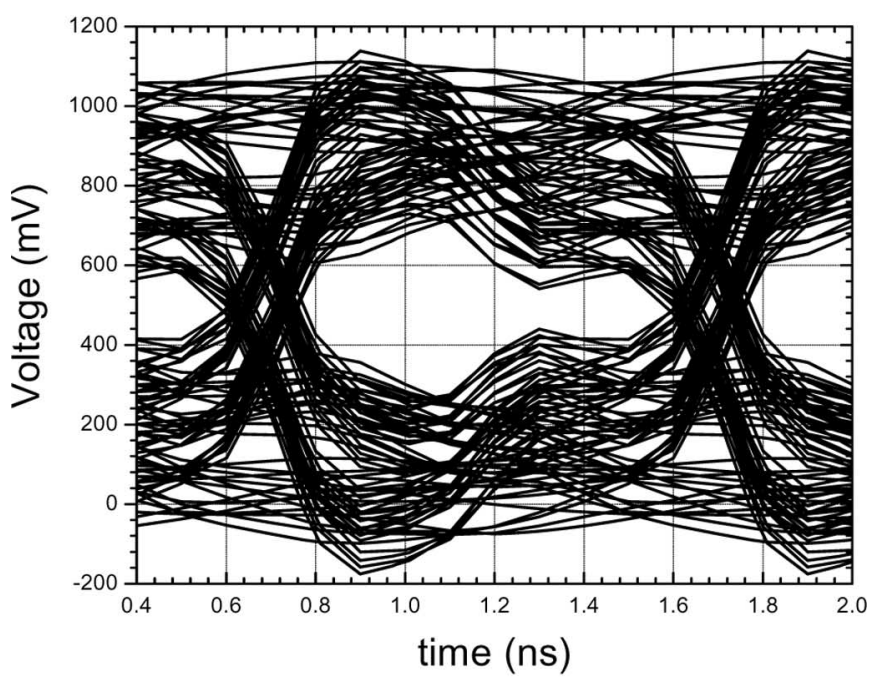

(b)

Fig. 12. Simulated eye diagram for single-ended trace. (a) Solid reference board. (b) Meander-L EBG-patterned reference board.

centers of the two lines is $3 \mathrm{~mm}$. Two parameters, maximum eye open (MEO) and maximum eye width (MEW), are used as metrics of the eye pattern quality. MEO represents the maximum difference between points in the interior of the eye diagram at the same time instant. MEW measures the maximum difference at the same voltage level between two points in the interior of the eye diagram. The simulated eye diagram in Fig. 13(b) shows for the differential signaling case, $\mathrm{MEO}=950 \mathrm{mV}$, and $\mathrm{MEW}=980 \mathrm{ps}$. This is a sizable improvement in comparison to that in the case of the single line.

\section{Eye Diagram for Supercell Structure}

If the Meander-L EBG-patterned surface is replaced with the supercell structure, the simulated eye diagram for a singleended signal is shown in Fig. 14. The test pattern is the same as that described earlier. It can be seen that for a supercell-based structure, $\mathrm{MEO}=800 \mathrm{mV}$, and $\mathrm{MEW}=940 \mathrm{ps}$. Comparing the results of Fig. 14 and Fig. 12(b), we observe that the eye pattern 


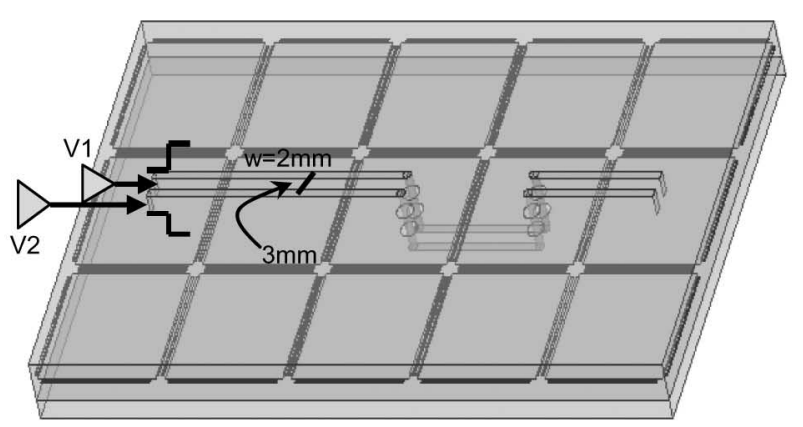

(a)

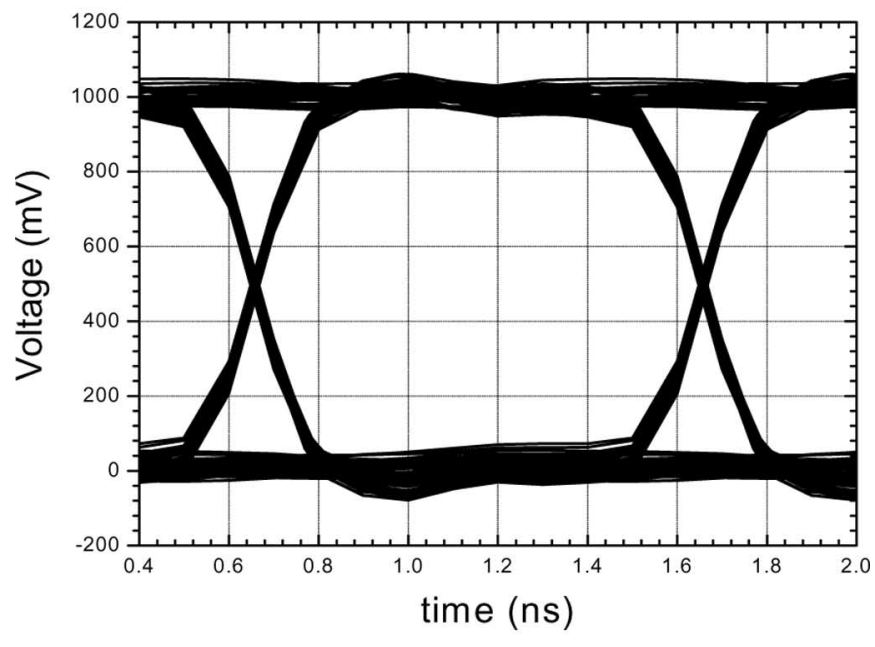

(b)

Fig. 13. (a) Test geometry for Meander-L EBG-patterned structure for differential pair configuration. (b) Corresponding eye diagram.

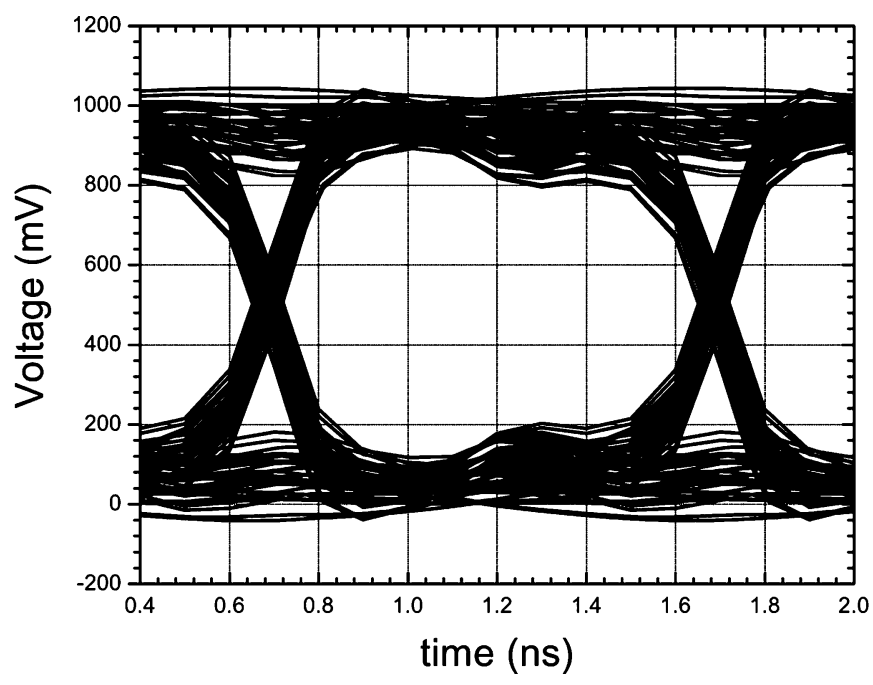

Fig. 14. Eye diagram for supercell EBG structure for the single-ended signal configuration.

for the supercell EBG-based structure is more open than the case of Meander- $\mathrm{L}$ for the single-ended configuration. A possible explanation for this is that the Meander-L structure induces extra phase difference per unit length (due to increased inductance) when the signal propagates along the traces, which results in the degradation of signal quality.

\section{CONCLUSION}

New types of planar EBG structures for suppression of power/ground noise propagation in high-speed circuits have been proposed. Simulation and measurement show that the EBG-patterned power plane incorporating meander lines as a dc link between patches can effectively achieve wideband noise mitigation. By periodically cascading two patches with different topology, an ultrawide bandgap for the suppression of SSN propagation extending from $250 \mathrm{MHz}$ to $12 \mathrm{GHz}$ is achieved. The radiation leakage is also studied numerically and experimentally. When comparison is made with the PCB with solid reference plane, the planar EBG structures introduced here can effectively suppress spurious radiation induced by the cavity modes of the PCB. In addition, it is found that the stronger field leakage from these perforated EBG structures is mainly concentrated around the feed point, and the field intensity attenuates as the distance from the source increases. These EBG-based structures can be used to further reduce EMI. Finally, the effect of the new planar EBG structures on SI is investigated. Using eye diagram simulation as a measure of signal quality, it is concluded that acceptable quality of data transmission at high-speed clock rate can be achieved with the supercell EBG-based structure, and signal quality can be further improved if differential signaling is used.

In summary, the proposed planar EBG structures not only strongly reduce the power/ground noise propagation for a wide frequency range, but also achieve broadband field mitigation for EMI applications.

\section{REFERENCES}

[1] L. D. Smith, "Simultaneous switching noise and power plane bounce for CMOS technology," in Proc. IEEE 8th Top. Meet. Electr. Perform. Electron. Packag., San Deigo, CA, Oct. 1999, pp. 163-166.

[2] R. Senthinatan and J. Price, Simultaneous Switching Noise of CMOS Devices and Systems. Norwell, MA: Kluwer, 1994.

[3] V. Ricchiuti, "Power-supply decoupling on fully populated high-speed digital PCBs," IEEE Trans. Electromagn. Compat., vol. 43, no. 4, pp. 671676, Nov. 2001.

[4] M. Xu, T. H. Hubing, J. Chen, T. P. Van Doren, J. L. Drewniak, and R. E. DuBroff, "Power-bus decoupling with embedded capacitance in printed circuit board design," IEEE Trans. Electromagn. Compat., vol. 45, no. 1, pp. 22-30, Feb. 2003.

[5] D. F. Sievenpiper, L. Zhang, R. F. J. Broag, N. G. Alexopolous, and E. Yablonovitch, "High-impedance electromagnetic surfaces with a forbidden frequency band," IEEE Trans. Microw. Theory Tech., vol. 47, no. 11, pp. 2059-2073, Nov. 1999.

[6] D. F. Sievenpiper, "High-impedance electromagnetic surfaces" Ph.D. dissertation, Dept. Elect. Eng, Univ. California Los Angeles, Los Angeles, CA, 1999.

[7] R. Abhari and G. V. Eleftheriades, "Metallo-dielectric electromagnetic bandgap structures for suppression and isolation of the parallel-plate noise in high-speed circuits," IEEE Trans. Microw. Theory Tech., vol. 51, no. 6, pp. 1629-1639, Jun. 2003.

[8] T. Kamgaing and O. M. Ramahi, "A novel power plane with integrated simultaneous switching noise mitigation capability using high impedance surface," IEEE Microw. Wireless Compon. Lett., vol. 13, no. 1, pp. 21-23, Jan. 2003.

[9] S. Shahparnia and O. M. Ramahi, "Miniaturized electromagnetic bandgap structures for broadband switching noise suppression in PCBs," Electron. Lett., vol. 41, no. 9, pp. 519-520, Apr. 2005.

[10] T. Kamgaing and O. M. Ramahi, "Inductance-enhanced high-impedance surfaces for broad-band simultaneous switching noise mitigation in power planes," in IEEE MTT-S Int. Microw. Symp. Dig., vol. 3, pp. 2165-2168, Jun. 2003. 
[11] S. D. Rogers, "Electromagnetic-bandgap layers for broad-band suppression of TEM modes in power planes," IEEE Trans. Microw. Theory Tech., vol. 53, no. 8, pp. 2495-2505, Aug. 2005.

[12] S. Shahparnia, B. Mohajer-Iravani, and O. M. Ramahi, "Electromagnetic noise mitigation in high-speed printed circuit boards and packaging using electromagnetic bandgap structures," in Proc. 54th Electron. Compon. Technol. Conf., Las Vegas, NV, Jun. 2004, pp. 1831-1836.

[13] F. R. Yang, K. P. Ma, and T. Itoh, "A uniplanar compact photonic-bandgap (UC-PBG) structure and its applications for microwave circuits," IEEE Trans. Microw. Theory Tech., vol. 47, no. 8, pp. 1509-1514, Aug. 1999.

[14] T. L. Wu, C. C. Yang, Y. H. Lin et al., "A novel power plane with superwideband elimination of ground bounce noise on high speed circuits," IEEE Microw Wireless Compon. Lett., vol. 15, no. 3, pp. 174-176, Mar. 2005.

[15] J. Qin and O. M. Ramahi, "Ultra-wideband mitigation of simultaneous switching noise using novel planar electromagnetic bandgap structure," IEEE Microw. Wireless Compon. Lett., vol. 16, no. 9, pp. 487-489, Sep. 2006.

[16] J. Choi, V. Govind, M. Swaminathan, L. Wan, and R. Doraiswami, "Isolation in mixed-signal systems using a novel electromagnetic bandgap (EBG) structure," in Proc. IEEE 13th Top. Meet. Electr. Perform. Electron. Packag., Portland, OR, Oct. 2004, pp. 199-202.

[17] T. L. Wu, Y. H. Lin, and S. T. Chen, "A novel power planes with low radiation and broadband suppression of ground bounce noise using photonic bandgap structures," IEEE Microw. Wireless Compon. Lett., vol. 14, no. 7, pp. 337-339, Jul. 2004.

[18] N. Shino and Z. Popovic, "Radiation from ground-plane photonic bandgap microstrip waveguide," IEEE MTT-S Int. Microw. Symp. Dig, vol. 2, pp. 1079-1082, Jun. 2002.

[19] J. Lee, H. Kim, and J. Kim, "High dielectric constant thin film EBG power/ground network for broad-band suppression of SSN and radiated emissions," IEEE Microw. Wireless Compon. Lett., vol. 15, no. 8, pp. 505507, Aug. 2005

[20] Y. Ko, K. Ito, J. Kudo, and T. Sudo, "Electromagnetic radiation properties of a printed circuit board with a slot in the ground plane," in Proc. Int. Symp. Electromagn. Compat., Tokyo, Japan, May1999, pp. 576-579.

[21] HFSS, High Frequency Structure Simulator version 9.2, Ansoft Corp. Pittsburgh, PA.

[22] Jianming Jin, The Finite Elements in Electromagnetics. New York: Wiley, 2002.

[23] T. H. Hubing, "Printed circuit board EMI source mechanisms," in Proc. IEEE Int. Symp. Electromagn. Compat., Boston, MA, Aug. vol. 1, 2003, pp. $1-3$.

[24] M. Leone, "The radiation of a rectangular power bus structure at multiple cavity-mode resonances," IEEE Trans. Electromagn. Compat., vol. 45, no. 3, pp. 486-492, Aug. 2003.

[25] N. Na, J. Choi, S. Chun, M. Swaminathan, and J. Srinivasan, "Modeling and transient simulation of planes in electronic packages," IEEE Trans. Adv. Packag., vol. 23, no. 3, pp. 340-352, Aug. 2000.

[26] P. Clayton, Introduction to Electromagnetic Compatibility. Hoboken, NJ: Wiley-Interscience, 1992.

[27] J. C. W. Ho, Q. Zhu, and R. Abhari, "Modeling of transmission lines with textured ground planes and investigation of data transmission by generating eye diagrams," in Proc. IEEE 13th Top. Meet. Electr. Perform. Electron. Packag., Portland, OR, Oct. 2004, pp. 195-198.

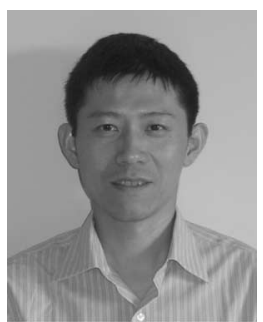

Jie Qin received the B.S. degree in electrical engineering from Zhejiang University, Hangzhou, China, in 1990, the M.S. degree in electrophysics from the Chinese Academy of Science, Beijing, in 1996, and the $\mathrm{Ph} . \mathrm{D}$. degree in electrical and computer engineering from the University of Maryland, College Park, in 2006 .

From 1990 to 1997, he was a Research Staff member in the Xian Institute of Optics and Precision Mechanics, Chinese Academy of Science. There he made contributions to several projects in developing new optoelectronic devices. He then joined the National Key Laboratory of Applied Surface Physics, Fudan University, Shanghai, China, where he carried on research on semiconductor microfabrication and nanoelectronics. His current research interests include electromagnetic bandgap structures, design of RF and microwave devices, high-speed packaging, and signal integrity.

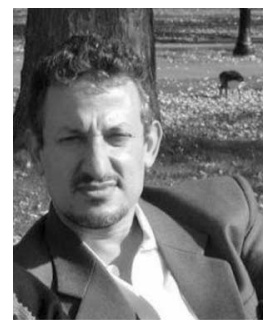

Omar M. Ramahi received the B.S. degree in mathematics and electrical and computer engineering (summa cum laude) from Oregon State University, Corvallis, and the M.S. and Ph.D. degrees in electrical and computer engineering from the University of Illinois at Urbana-Champaign, Champaign.

From 1990 to 1993, he was a Visiting Fellow with the University of Illinois at Urbana-Champaign. From 1993 to 2000, he was with the Digital Equipment Corporation (presently Hewlett-Packard), Maynard, MA, where he was a member of the Alpha Server Product Development Group. In August 2000, he joined the faculty of the James Clark School of Engineering, University of Maryland, College Park, where he was also a Faculty member of the CALCE Electronic Products and Systems Center. He is currently the Associate NSERC/RIM Industrial Research Chair in the Electrica and Computer Engineering Department, University of Waterloo, Waterloo, ON, Canada. He has been a Consultant to several companies and was a cofounder of EMS-PLUS, Four Oaks, NC, and Applied Electromagnetic Technology, LLC, Solomons, MD. His research interests include experimental and computational EMI/EMC studies, high-speed devices and interconnects, biomedical applications of electromagnetics, novel optimization techniques, and interdisciplinary studies linking electromagnetic application to novel materials. He has authored and coauthored over 150 papers published in several journals and conference proceedings, and has coauthored EMI/EMC Computational Modeling Handbook, 2nd ed. (Springer-Verlag, 2001).

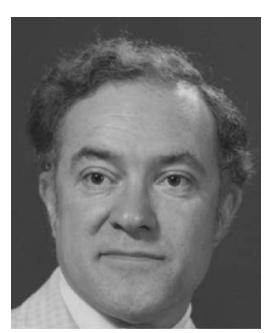

Victor L. Granatstein (S'59-M'64-SM'86-F'92LF'02) received the Ph.D. degree in electrical engineering from Columbia University, New York, in 1963.

From 1964 to 1972, he was a Research Scientist with Bell Telephone Laboratories, Murray Hill, NJ, after a year of postdoctoral work at Columbia University. He was a Visiting Senior Lecturer with the Hebrew University of Jerusalem, Jerusalem, Israel, in 1969-1970. In 1972, he joined the Naval Research Laboratory (NRL), Washington, DC, as a Research Physicist, where he was the Head of NRL's High Power Electromagnetic Radiation Branch from 1978 to 1983. Since August 1983, he has been a Professor in the Electrical and Computer Engineering Department, University of Maryland, College Park. From 1987 to1998, he was the Director of the Institute for Plasma Research (now renamed Institute for Research in Electronics and Applied Physics), University of Maryland. He was a Visiting Professor at Tel Aviv University, Tel Aviv, Israel, in 1994 and 2003, where he became the Sackler Professor by Special Appointment in 2004. His current research interests include electromagnetic radiation from relativistic electron beams, advanced concepts in millimeter wave tubes, and the effects of high-power microwaves on electronic circuits and systems. He has coauthored more than 250 research papers in several scientific journals and has coedited three books. He holds a number of patents on active and passive microwave devices.

Dr. Granatstein is a Fellow of the American Physical Society. He has received a number of major research awards including the E.O. Hulbert Annual Science Award in 1979, the Superior Civilian Service Award in 1980, the Captain Robert Dexter Conrad Award for scientific achievement awarded by the Secretary of the Navy in 1981, the IEEE Plasma Science and Applications Award in 1991, and the Robert L. Woods Award for Excellence in Electronics Technology in 1998. 